\title{
The Mediation Effect of Customer Perceived Value and AttitudeToward Advertisement on Social Media Influencer's Credibility on Purchase Intention
}

\author{
Liem Pamela Lukito ${ }^{1)}$ \\ Retno Yustini ${ }^{2)}$ \\ 1) Student of Master of Management Soegijapranata \\ Catholic University Semarang \\ 2) Lecturer of Soegijapranata Catholic University Semarang \\ Email: pamelalukito@yahoo.com /ph: 082134627772
}

\begin{abstract}
Instagram isthe most used social mediathat promotes influencer marketing, which is an effective marketing way by using influencer. SMI can be a fashion blogger, such as Gisella who endorses casual fashion in Instagram. SMI's credibilityhas a role in purchase intention, making a producer consider SMI's credibility when they decide to work with them. Customer perceived value (CPV) can be partially mediating variable toward SMI's credibility on purchase intention, indicating that there might be other variable that act as mediator. Other researcher found that attitude toward advertisement (Aad) act as mediator of SMI's credibility on purchase intention. Thus the purpose of this research is to examine the mediating effect of CPV and Aad toward the SMI's credibility on purchase intention of casual fashion. The population used was female student of SCU Semarang. Descriptive analysis (score tabulation) and statistic analysis (regression, path analysis)were conducted. The result indicated that CPV and Aad are partially mediating SMI's credibility on purchase intention of casual fashion endorsed by Gisella. It implied that producer should screen SMI based on their credibility since it has a great direct effect on purchase intention. Furthermore, they should consider CPV and Aad since it poses a mediating role.
\end{abstract}

Keywords: attitude toward advertisement, credibility, customer perceived value, social media influencer, purchase intention.

\section{INTRODUCTION}

In this globalization era, the number of internet user increased. According to Smartinsights (2016), the increasing number reached 14 percent by 2015. Specifically, in Indonesia more than half of its population (51,5 percent) already used internet (Isparmo SEO, 2016). Normally, they spent eight hours 44 minutes each day to access internet. Moreover, 87,13 percent Indonesian people use internet to access social media (APJII, 2017 in DailySocialID, 2017). One of many social media which is mostly accessed by Indonesian 
people is Instagram. According to the research conducted by Isosui (2016), most respondent said they liked Instagram than other social media. Instagram itself is known to be the most used social media to conduct influencer marketing (Sociabuzz, 2018).

Influencer marketing is known to be an effective way to help one to achieve their marketing goals. Producer mainly uses this technique in order to improve brand awareness (98,8 percent), educate target (62,7 percent), improve sales (50,6percent), gain more followers (39,8percent), and search engine optimization (25,3percent). Influencer marketing technique mainly uses social media influencer (SMI) to help communicate and build relationship with consumer (Freberget al., 2011).

Social media influencer is a well-known person which can affect consumer's attitude (Szmigin and Piacentini, 2015), due to their attractiveness, expertise, and authenticity to create aesthetic content of product they endorsed (Burgess, 2017 in Gashi, 2017). Moreover, by choosing an appropriate SMI will help producer to position their product and alter mispositioned product.

One of many forms of SMI is known as fashion blogger. This kind of SMI succeeded to promote lifestyle product, such as fashion, through interacting with consumer, and also with their authenticity, confidence, and interactive (Sudha and Sheena, 2017). In Indonesia, fashion is the first product that is categorized as popular product in social media and online sites (JarvisStore, 2016) and categorized as the most bought product by online consumer (APJII, 2014).

Gisella Anastasia who is a well-known SMI, acts as fashion blogger. She endorsed mainly casual-type fashion. According to Instagram (2018), Gisella has 19,1 million followers, which LineToday (2018) claimed that Gisella is a feminist who is known to wear simple and chic fashion, such as casual fashion. Through her recommendation, consumer will be convinced that the products she endorsed is a must-have items. 
According to research conducted by Harly and Octavia (2014), Shimp (2003), Maharani and Totoatmojo (2015), Shafiq (2011), Indraswari and Pramudana (2014) can be concluded that SMI has a significant effect on purchase intention. Moreover, Gyda (2017) and Rochmania and Prabowo (2016) explained that SMI's credibility is used as a reference to producer that want to collaborate with them. Therefore in this research, SMI's credibility will be an independent variable, due to its importance in affecting purchase intention.

Besides, in this research, will also be explained the mediating role of customer perceived value toward the relationship between SMI's credibility and purchase intention. As researched by Arsinta and Purnami (2015) and Hanusdoh (2012) they concluded that customer perceived value can act as mediating variable toward SMI's credibility on purchase intention. However, in their research, Shafiq(2011) found that customer perceived value doesn't act as mediating variable toward SMI’s credibility on purchase intention.

Research conducted by Arsinta and Purnami (2015) concluded that customer perceived value poses partial mediation on SMI's credibility on purchase intention. Thus, in this research, attitude toward advertisement will be added as mediating variable on the influence of SMI's credibility on purchase intention. As mentioned by Stephanie (2013) who found that attitude toward advertisement act as mediating variable on the influence of SMI's credibility on purchase intention.

To fill this gap, researchers will explore the relationship between SMI's credibility and purchase intention, with customer perceived value as mediating variable. Casual fashion will be the object of this research, and the respondent will be female student of Soegijapranata Catholic University Semarang. The respondent chosen in this research is based on research conducted, that concluded 18-25 years old Indonesian are the most ones who access Instagram daily. 


\section{LITERATURE REVIEW}

\section{Social Media Influencer's Credibility}

Social Media Influencer (SMI) is individual who is popular in social media due to their charism, credibility, and good reputation. They are mainly attractive. According to Gyda (2017) SMI's credibility is used as a reference when a firm want to make partnership with SMI. Neveset al. (2015) continued, for a firm, things that need to be considered when choosing SMI as their partner are total post, total followers, and also the purpose of the firm itself to use SMI. Stafford et al. (2003) also described that influencer's credibility is positive characteristic that influence consumer's behavior. To measure SMI's credibility, indicators such as attractiveness, trustworthiness, expertise (Pakpahan, 2017), popular (Hansudoh, 2012), and suitable to promote certain product (Wangsa and Ardani, 2015) will be used in this research.

\section{Customer Perceived Value}

Customer perceived value is known as overall consumer's judgement on product based on perception they receive (Zeithaml, 1988 in Asgarpouret al., 2015). McDougall and Levesque (2000) continued that CPV is the result from benefit consumer gets, which is cost that consumer incurred when they purchase a product. Cost of the product isn't always monetary, but can also be non-monetary, such as time, experience, and product evaluation. To measure CPV, some indicators can be used such as (1) price is worth with product's value, (2) product is valueable for consumer, (3) product has a higher value than other products that celebrity endorsed (Arsinta and Purnami, 2015), (4) quality products, (5) convenience to get the product (Hansudoh, 2012). 


\section{Attitude Toward Advertisement}

Attitude is individual's tendency as a response to product offering, which can be good or not good (Alunat and Ariyanti, 2016). Dominanto (2007) contined that attitude toward advertisement is individual's tendency to evaluate certain advertisement in a positive or negative way. To measure attitude toward advertisement, indicators that can be used are (1) consumer likes the advertisement starring celebrity (Schiffman and Kanuk, 2010), (2) consumer evaluates the advertisement starring celebrity as an attractive advertisement, (3) consumer evaluates the advertisement starring celebrity as a good advertisement (Wijaya, 2015), (4) advertisement is easy to understand, and (5) advertisement is informative (Wangsa and Ardani, 2015).

\section{Purchase Intention}

Purchase intention is defined as consumer's desire to buy a product (Setiawan and Ihwan, 2004 in Ambarwatiet al., 2015). Some factors affecting online purchase intention are consumer's characteristic, website characteristic, store characteristic and product characteristic (Ari and Nasir, 2014). To measure purchase intention, indicators that can be used are (1) consumer wants to know more about product, (2) consumer considers to buy a product (Arsinta and Purnami, 2015), (3) consumer wants to buy a product that is endorsed by celebrity (Hansudoh, 2012), and (4) consumer refers the product celebrity endorsed (Ferdinand, 2002).

\section{METHODOLOGY}

This research was conducted using quantitative analysis that used purposive sampling as one of non-random sampling method. By using purposive sampling, it means that not all populations have the same chance to be chosen as respondent (Sugiyono, 2013). Population 
used in this research was female student of Soegijapranata Catholic University Semarang who (1) minimum 18 years old, (2) have Instagram account, (3) following Gisella Anastasia (@Gisel_la) in Instagram. Total sample used in this research referred to Hair et al., (2004) who concluded that in multivariate analysis, sample required is 100-200 respondent. Therefore in this research, 100 respondents with criteria above will be used.

Data collected by using questionnaire with 5 likert scale, which are $1=$ strongly disagree, 2 = disagree, 3 = neutral, 4 = agree, 5 = strongly agree. Questionnaire was spread during February 2019.

Validity and reliability of indicators and variables were conducted as measurement test. Validity test was conducted using bivariate correlation technique, referred to Ghozali (2011). Reliability test was conducted by observing Cronbach's Alpha value. Table 1., showed that cronbach's alpha value is 0,909. According to Ghozali (2011), this value showed that variables used in this research is reliable (value $>0,6$ ). Table 2., also showed that there are significant correlations between each indicator to total construct score. Therefore it can be said that all indicators used are valid.

Table 1. Reliability Test of Variables

\begin{tabular}{|c|c|}
\hline Cronbach's Alpha & N of Items \\
\hline 0,909 & 19 \\
\hline
\end{tabular}

Table 2. Validity Test of Each Indicator

\begin{tabular}{|c|c|}
\hline Indicator & $\begin{array}{c}\text { Correlation to Total } \\
\text { Construct Score }\end{array}$ \\
\hline KS1 & Significant \\
\hline KS2 & Significant \\
\hline KS3 & Significant \\
\hline KS4 & Significant \\
\hline KS5 & Significant \\
\hline CPV1 & Significant \\
\hline
\end{tabular}


*) KS = SMI's Credibility

\begin{tabular}{|c|c|}
\hline Indicator & $\begin{array}{c}\text { Correlation to Total } \\
\text { Construct Score }\end{array}$ \\
\hline CPV2 & Significant \\
\hline CPV3 & Significant \\
\hline CPV4 & Significant \\
\hline CPV5 & Significant \\
\hline SK1 & Significant \\
\hline SK2 & Significant \\
\hline SK3 & Significant \\
\hline SK4 & Significant \\
\hline SK5 & Significant \\
\hline MB1 & Significant \\
\hline MB2 & Significant \\
\hline MB3 & Significant \\
\hline MB4 & \\
\hline
\end{tabular}

$\mathrm{CPV}=$ Customer Perceived Value

SK = Consumer's attitude on Advertisement

$\mathrm{MB}=$ Purchase Intention

In this research, descriptive analysis and statistic were conducted. Descriptive analysis used in this research were score tabulation which explained how respondents respond to variables and indicators used in this research, which are SMI's credibility, customer perceived value, consumer's attitude toward advertisement, and purchase intention. Regression analysis will be conducted as a part of statistic test, to examine the influence of purchase intention's predictors which are SMI's credibility, customer perceived value, attitude toward advertisement. Path analysis and casual step method (Baron and Kenny, 1986) will be conducted to examine the influence of customer perceived value and attitude toward advertisement as mediating variables. Normality, multicolinerity and linearity test were conducted as those tests are required for path analysis. Here are the regression equations in this research:

(1) $\mathrm{CPV}=\alpha+\beta_{2} \mathrm{KS}+\mathrm{e} 1$

(2) $\mathrm{SK}=\alpha+\beta_{4} \mathrm{KS}+\mathrm{e} 2$ 


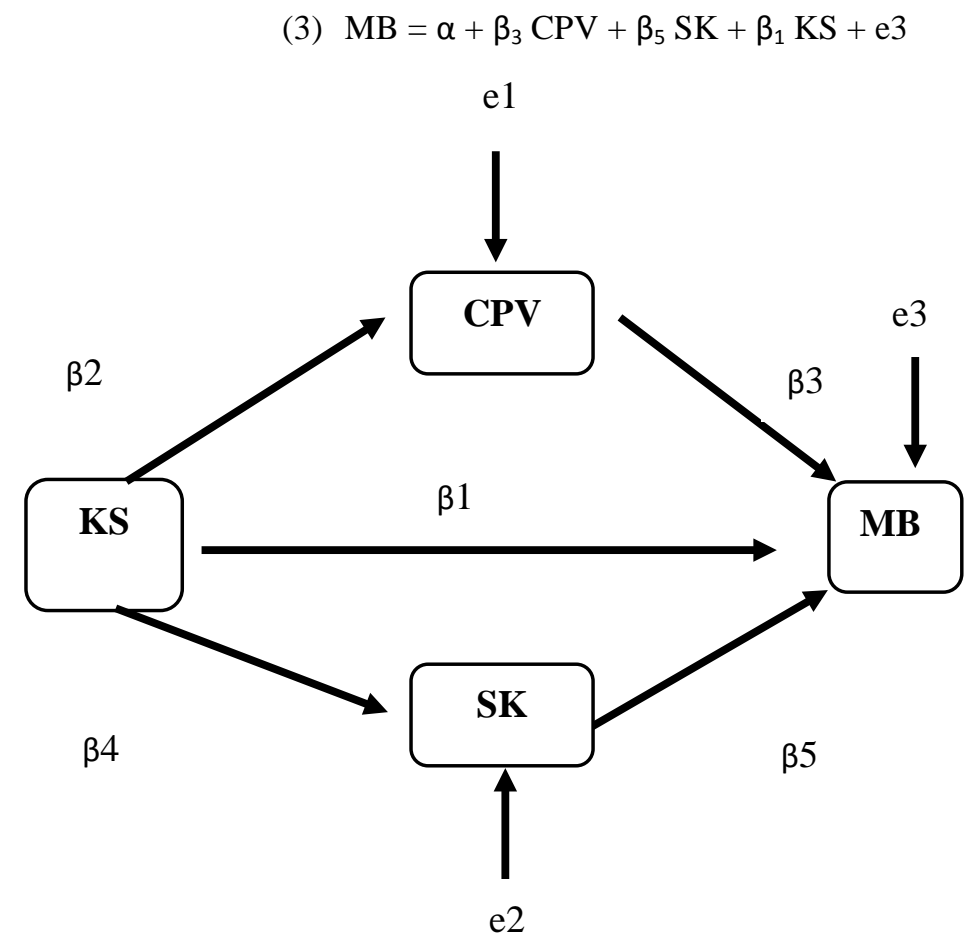

$=$ other factors influencing customer perceived value

$\begin{array}{ll}\text { e2 } & \text { = other factors influencing attitude toward advertisement } \\ \text { e3 } & =\text { other factors influencing purchase intention }\end{array}$

\section{RESULT AND DISCUSSION}

\section{Respondent Profile}

Respondents used in this research were female students of Soegijapranata Catholic University Semarang aged 18 or above and they are all followers of Gisella(@Gisel_la) in Instagram. Respondents came from business, food technology, psychology, law, and architecture faculty which most of them were major in business faculty. They mainly access Instagram one to three hours per day and usually see SMI's content each time they access their Instagram account. They checked SMI's account in order to see SMI's content due to 
their aesthetic posts. Then, most of respondents said that they ever made at least one to two online purchase each month.

\section{Score Tabulation}

Table 3., showed how respondents responded to each indicator of each variable used in this research, such as SMI's credibility, customer perceived value, attitude toward advertisement, and purchase intention.

Table 3. Score Tabulation

\begin{tabular}{|c|c|c|c|c|c|c|c|c|}
\hline \multirow{2}{*}{$\begin{array}{l}\mathrm{N} \\
\mathrm{o}\end{array}$} & \multirow{2}{*}{ Indicators } & \multicolumn{5}{|c|}{ Answer’s Frequency (Score) } & \multirow{2}{*}{$\begin{array}{l}\text { Avera } \\
\text { ge }\end{array}$} & \multirow{2}{*}{$\begin{array}{l}\text { Informati } \\
\text { on }\end{array}$} \\
\hline & & $\begin{array}{c}\mathrm{ST} \\
\mathrm{S}\end{array}$ & TS & $\mathrm{N}$ & $\mathrm{S}$ & SS & & \\
\hline \multicolumn{9}{|c|}{ SMI’s Credibility } \\
\hline 1. & Gisellamemilikidaya Tarik & $\begin{array}{c}0 \\
(0)\end{array}$ & $\begin{array}{c}5 \\
(10 \\
(10\end{array}$ & $\begin{array}{c}33 \\
(99)\end{array}$ & $\begin{array}{l}42 \\
(16 \\
8)\end{array}$ & $\begin{array}{c}20 \\
(10 \\
0)\end{array}$ & 3,77 & Agree \\
\hline 2. & Giselladapatdipercaya & $\begin{array}{c}0 \\
(0)\end{array}$ & $\begin{array}{c}5 \\
(10 \\
(10\end{array}$ & $\begin{array}{c}62 \\
(18 \\
6)\end{array}$ & $\begin{array}{l}27 \\
(10 \\
8)\end{array}$ & $\begin{array}{c}6 \\
(30)\end{array}$ & 3,34 & Neutral \\
\hline 3. & $\begin{array}{l}\text { Gisellaahlimemadupadankanpakaian } \\
\text { casual }\end{array}$ & $\begin{array}{c}1 \\
(1)\end{array}$ & $\begin{array}{c}2 \\
(4)\end{array}$ & $\begin{array}{c}25 \\
(75)\end{array}$ & $\begin{array}{c}60 \\
(24 \\
0)\end{array}$ & $\begin{array}{c}12 \\
(60)\end{array}$ & 3,8 & Agree \\
\hline 4. & $\begin{array}{l}\text { Gisellamerupakanselebgram popular yang } \\
\text { dikenalbanyak orang }\end{array}$ & $\begin{array}{c}1 \\
(1)\end{array}$ & $\begin{array}{c}3 \\
3 \\
(6)\end{array}$ & $\begin{array}{c}28 \\
(84)\end{array}$ & $\begin{array}{l}48 \\
(19 \\
2)\end{array}$ & $\begin{array}{l}20 \\
(10 \\
0)\end{array}$ & 3,83 & Agree \\
\hline 5. & $\begin{array}{l}\text { Gisellacocokdalammelakukan endorse } \\
\text { pakaian casual }\end{array}$ & $\begin{array}{c}0 \\
(0)\end{array}$ & $\begin{array}{c}2 \\
(4)\end{array}$ & $\begin{array}{c}33 \\
(99)\end{array}$ & $\begin{array}{l}54 \\
(21 \\
6)\end{array}$ & $\begin{array}{c}11 \\
(55)\end{array}$ & 3,74 & Agree \\
\hline \multicolumn{7}{|c|}{ Total Score Average } & 3,69 & Agree \\
\hline \multicolumn{9}{|c|}{ Customer Perceived Value } \\
\hline 6. & $\begin{array}{l}\text { Pakaian casual yang di-endorse } \\
\text { Gisellabermanfaat }\end{array}$ & $\begin{array}{c}6 \\
(6)\end{array}$ & $\begin{array}{c}24 \\
(48 \\
)\end{array}$ & $\begin{array}{c}55 \\
(16 \\
5)\end{array}$ & $\begin{array}{c}13 \\
(52)\end{array}$ & $\begin{array}{c}2 \\
(10)\end{array}$ & 2,81 & Neutral \\
\hline 7. & $\begin{array}{l}\text { Pakaian casual yang di-endorse } \\
\text { Gisellamemilikiharga yang } \\
\text { sesuaidenganmanfaat yang didapatkan }\end{array}$ & $\begin{array}{c}0 \\
(0)\end{array}$ & $\begin{array}{c}14 \\
(28 \\
)\end{array}$ & $\begin{array}{c}62 \\
(18 \\
6) \\
\end{array}$ & $\begin{array}{c}23 \\
(92)\end{array}$ & $\begin{array}{c}1 \\
(5)\end{array}$ & 3,11 & Neutral \\
\hline 8. & $\begin{array}{l}\text { Gisellamampumeningkatkannilaipakaian } \\
\text { casual }\end{array}$ & $\begin{array}{c}0 \\
(0)\end{array}$ & $\begin{array}{c}22 \\
(44 \\
)\end{array}$ & $\begin{array}{c}62 \\
(18 \\
6)\end{array}$ & $\begin{array}{c}15 \\
(60)\end{array}$ & $\begin{array}{c}1 \\
(5)\end{array}$ & 2,95 & Neutral \\
\hline 9. & $\begin{array}{l}\text { KualitasPakaian casual yang di-endorse } \\
\text { Gisellasebagai SMI } \\
\text { berkualitasinternasional }\end{array}$ & $\begin{array}{c}1 \\
(1)\end{array}$ & $\begin{array}{c}22 \\
(44 \\
)\end{array}$ & $\begin{array}{l}62 \\
(18 \\
6) \\
\end{array}$ & $\begin{array}{c}13 \\
(52)\end{array}$ & $\begin{array}{c}2 \\
(10)\end{array}$ & 2,93 & Neutral \\
\hline 10 & $\begin{array}{l}\text { Pakaian casual yang di-endorse } \\
\text { Gisellasebagai SMI mudahdidapatkan }\end{array}$ & $\begin{array}{c}0 \\
(0)\end{array}$ & $\begin{array}{c}1 \\
(2)\end{array}$ & $\begin{array}{c}49 \\
(14 \\
7)\end{array}$ & $\begin{array}{c}42 \\
(16 \\
8)\end{array}$ & $\begin{array}{c}8 \\
(40)\end{array}$ & 3,57 & Agree \\
\hline \multicolumn{7}{|c|}{ Total Score Avera } & 3,07 & Neutral \\
\hline
\end{tabular}




\begin{tabular}{|c|c|c|c|c|c|c|c|c|}
\hline \multirow{2}{*}{$\begin{array}{l}\mathrm{N} \\
\mathrm{o}\end{array}$} & \multirow[t]{2}{*}{ Indicators } & \multicolumn{5}{|c|}{ Answer’s Frequency (Score) } & \multirow{2}{*}{$\begin{array}{l}\text { Avera } \\
\text { ge }\end{array}$} & \multirow{2}{*}{$\begin{array}{l}\text { Informat } \\
\text { on }\end{array}$} \\
\hline & & $\begin{array}{l}\text { ST } \\
\text { S }\end{array}$ & TS & $\mathrm{N}$ & $\mathrm{S}$ & SS & & \\
\hline \multicolumn{9}{|c|}{ Attitude Toward Advertisement } \\
\hline 11 & $\begin{array}{l}\text { Iklanpakaian casual yang } \\
\text { dibintangiGisellasebagai SMI, disukai }\end{array}$ & $\begin{array}{c}0 \\
(0)\end{array}$ & $\begin{array}{c}5 \\
(10 \\
)\end{array}$ & $\begin{array}{l}59 \\
(17 \\
7)\end{array}$ & $\begin{array}{c}34 \\
(13 \\
6)\end{array}$ & $\begin{array}{c}2 \\
(10)\end{array}$ & 3,33 & Neutral \\
\hline 12 & $\begin{array}{l}\text { Iklanpakaian casual yang } \\
\text { dibintangiGisellasebagai SMI, } \\
\text { merupakaniklan yang menarik }\end{array}$ & $\begin{array}{c}0 \\
(0)\end{array}$ & $\begin{array}{c}5 \\
(10 \\
)\end{array}$ & $\begin{array}{l}52 \\
(15 \\
6)\end{array}$ & $\begin{array}{l}40 \\
(16 \\
0)\end{array}$ & $\begin{array}{c}3 \\
(15)\end{array}$ & 3,41 & Agree \\
\hline 13 & $\begin{array}{l}\text { Iklanpakaian casual yang } \\
\text { dibintangiGisellasebagai SMI, } \\
\text { merupakaniklan yang bagus }\end{array}$ & $\begin{array}{c}0 \\
(0)\end{array}$ & $\begin{array}{c}3 \\
(6)\end{array}$ & $\begin{array}{c}54 \\
(16 \\
2)\end{array}$ & $\begin{array}{l}39 \\
(15 \\
6) \\
\end{array}$ & $\begin{array}{c}4 \\
(20)\end{array}$ & 3,44 & Agree \\
\hline 14 & $\begin{array}{l}\text { Iklanpakaian casual yang } \\
\text { dibintangiGisellasebagai SMI, } \\
\text { merupakaniklan yang mudahdimengerti }\end{array}$ & $\begin{array}{c}0 \\
(0)\end{array}$ & $\begin{array}{c}2 \\
(4)\end{array}$ & $\begin{array}{l}42 \\
(12 \\
6) \\
\end{array}$ & $\begin{array}{c}54 \\
(21 \\
6) \\
\end{array}$ & $\begin{array}{c}4 \\
(20)\end{array}$ & 3,62 & Agree \\
\hline 15 & $\begin{array}{l}\text { Iklanpakaian casual yang } \\
\text { dibintangiGisellasebagai SMI, } \\
\text { merupakaniklan yang informatif }\end{array}$ & $\begin{array}{c}1 \\
(1)\end{array}$ & $\begin{array}{c}4 \\
(8)\end{array}$ & $\begin{array}{l}50 \\
(15 \\
0)\end{array}$ & $\begin{array}{c}42 \\
(16 \\
8)\end{array}$ & $\begin{array}{c}3 \\
(15)\end{array}$ & 3,42 & Agree \\
\hline \multicolumn{7}{|c|}{ Total Score Average } & 3,44 & Agree \\
\hline \multicolumn{9}{|c|}{ Purchase Intention } \\
\hline 16 & $\begin{array}{l}\text { Sayainginmengetahuilebihdalamakanprod } \\
\text { ukpakaian casual yang di-endorse Gisella }\end{array}$ & $\begin{array}{c}3 \\
(3)\end{array}$ & $\begin{array}{c}26 \\
(52 \\
)\end{array}$ & $\begin{array}{l}45 \\
(13 \\
5)\end{array}$ & $\begin{array}{c}24 \\
(96)\end{array}$ & $\begin{array}{c}2 \\
(10)\end{array}$ & 2,96 & Neutral \\
\hline 17 & $\begin{array}{l}\text { Sayamempertimbangkanuntukmembeliprod } \\
\text { ukpakaian casual yang di-endorse Gisella }\end{array}$ & $\begin{array}{c}1 \\
(1)\end{array}$ & $\begin{array}{c}16 \\
(32 \\
)\end{array}$ & $\begin{array}{l}43 \\
(12 \\
9)\end{array}$ & $\begin{array}{l}34 \\
(13 \\
6) \\
\end{array}$ & $\begin{array}{c}6 \\
(30)\end{array}$ & 3,28 & Neutral \\
\hline 18 & $\begin{array}{l}\text { Sayainginmembelipakaian casual karena } \\
\text { di-endorse Gisella }\end{array}$ & $\begin{array}{c}3 \\
(3)\end{array}$ & $\begin{array}{c}30 \\
(60 \\
)\end{array}$ & $\begin{array}{l}47 \\
(14 \\
1)\end{array}$ & $\begin{array}{c}19 \\
(76)\end{array}$ & $\begin{array}{c}1 \\
(5)\end{array}$ & 2,85 & Neutral \\
\hline 19 & $\begin{array}{l}\text { Sayamereferensikanpakaian casual yang } \\
\text { di-endorse Gisella }\end{array}$ & $\begin{array}{c}3 \\
(3)\end{array}$ & $\begin{array}{c}30 \\
(60 \\
)\end{array}$ & $\begin{array}{l}49 \\
(14 \\
7) \\
\end{array}$ & $\begin{array}{c}16 \\
(64)\end{array}$ & $\begin{array}{c}2 \\
(10)\end{array}$ & 2,84 & Neutral \\
\hline \multicolumn{7}{|c|}{ Total Score Average } & 2,98 & Neutral \\
\hline
\end{tabular}

From table 3., above we can see that in variable SMI's credibility, KS 2 has the smallest score. This indicates that Gisella as SMI should improve consumer's trust through honest review of casual fashion. Gisella can also gain consumer's trust by wearing casual fashion she recommends. This way consumer may see that Gisella wears and enjoys casual fashion she recommends.

Indicator in customer perceived value that need to be considered since it has the smallest score is CPV 6. This indicates that Gisella should share what can be the benefit by wearing casual fashion she recommends, such as comfortable, great design etc. 
Then, indicator in attitude toward advertisement that has the smallest score is SK 11. This result showed that Gisella should pay attention to advertisement she makes. Gisella can combine some advertisement components, such as background color, lighting, and also using video to promote the product, in order to make consumer likes the advertisement she does.

Gisella also should consider indicator MB 19 which implies that Gisella should convince consumer that casual fashion she endorses is worth to buy, so they want to refer it to their environment. Gisella can also emphasize aesthetic content through her post, as in the descriptive analysis we found that female students checked SMI's account in order to see their aesthetic content.

Overall, it also can be concluded from table 3., that purchase intention variable has the smallest total average score. This implies that Gisella should be more creative and more aggressive to attract consumers in order to improve purchase intention.

\section{Normality Test}

Normality test was conducted to examine whether data is normally distributed. In this research, Kolmogorov-Smirnov test was used. Data is normally distributed if sig. value 0,05 (Ghozali 2011). From table 4., it can be concluded that all variables were all normally distributed.

Table 4. Normality Test

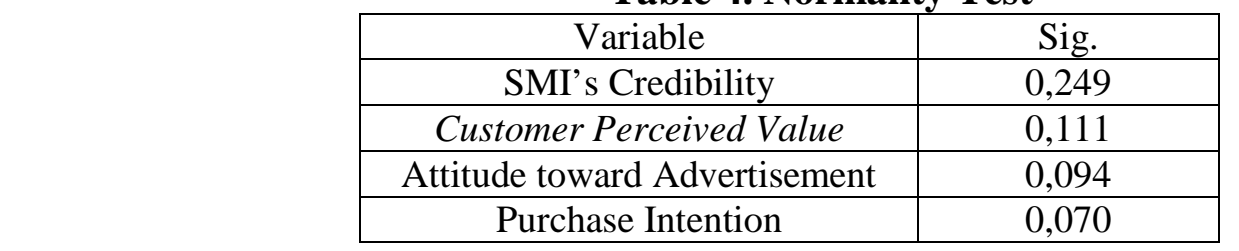

\section{Multicolinerity Test}

Multicolinerity test was used to examine whether there's correlation on independent variables in regression model. If there's correlation, there will be multicolinerity problem. In this research, we examine multicolinerity by observing VIF and tolerance value. Regression 
model doesn't posemulticolinerity if VIF value is below 10 and tolerance value is more than

0,1 (Ghozali, 2011).

Table 5. Multicolinerity Test

\begin{tabular}{|c|c|c|}
\hline Variable & \multicolumn{2}{|c|}{ Colinerity Statistic } \\
\hline SMI’s Credibility & Tolerance & VIF \\
\hline Customer Perceived Value & 0,645 & 1,551 \\
\hline $\begin{array}{c}\text { Attitude toward } \\
\text { Advertisement }\end{array}$ & 0,664 & 1,506 \\
\hline Purchase Intention & 0,645 & 1,551 \\
\hline
\end{tabular}

From table 5., above we can see that all variables doesn't pose multiolinerity problem, which can be observed through VIF value below 10 and tolerance value more than 0,1.

\section{Linearity Test}

Linearity test was conducted to examine whether data is distributed linearly. In this research, linearity was observed through deviation from linearity significance value with criteria, if significance value is above 0,05, data is linear. From table 6., below it can be observed that all variables' significance values are above 0,05 which means the data are linear.

Table 6. Linearity Test

\begin{tabular}{|c|c|}
\hline Variable & Sig. \\
\hline SMI's Credibility*Purchase Intention & 0,875 \\
\hline $\begin{array}{c}\text { Customer Perceived Value*Purchase } \\
\text { Intention }\end{array}$ & 0,580 \\
\hline $\begin{array}{c}\text { Atitude toward } \\
\text { Advertisement*Purchase Intention }\end{array}$ & 0,057 \\
\hline
\end{tabular}

$\mathbf{R}^{2}$

$\mathrm{R}^{2}$ test was conducted to examine model's ability to explain variation of dependent variables. $\mathrm{R}^{2}$ value that close to 1 , explain that independent variables used in this research have almost all information to predict dependent variable (Ghozali, 2011). From table 7., it can be seen that $\mathrm{R}^{2}$ value is 0,515 . This result implies that purchase intention is 51,5 percent 
influenced by SMI's credibility, customer perceived value, and attitude toward advertisement. There are other variables (48,5 percent) that influence purchase intention, which is not included in this research.

Table 7. $\mathbf{R}^{2}$ Test

\begin{tabular}{|c|c|}
\hline Mode & R Square \\
\hline 1 & 0,515 \\
\hline
\end{tabular}

\section{Regression Analysis}

Regression analysis used to examine the influence of SMI’s credibility on customer perceived value, SMI's credibility on attitude toward advertisement, SMI's credibility on purchase intention, customer perceived value on purchase intention, attitude toward advertisement on purchase intention.

Table 8. Influence of SMI's Credibility on Customer Perceived Value

\begin{tabular}{|c|c|c|c|c|c|}
\hline \multirow{2}{*}{ Variabel } & \multicolumn{2}{|c|}{$\begin{array}{c}\text { Unstandardzised } \\
\text { coefficients }\end{array}$} & $\begin{array}{c}\text { Standardized } \\
\text { coefficients }\end{array}$ & \multirow{2}{*}{ Signifikansi } \\
\cline { 2 - 4 } & $\mathrm{B}$ & Std. Error & Beta & & \\
\hline Constant & 7,129 & 1,433 & & & \\
\hline Kredibilitas SMI & 0,446 & 0,077 & 0,507 & 5,816 & 0,000 \\
\hline
\end{tabular}

Table 8., showed that significance value of the influence of SMI's credibility on customer perceived value is 0,000 or below 0,05 . This implies that Gisella's credibility as SMI influence customer perceived value toward casual fashion. This result is in line with research conducted by Hansudoh (2012), Harly and Octavia (2014), and Arsinta and Purnami (2015) who found that SMI's credibility influence customer perceived value.

Table 9. Influence of SMI's Credibility on Attitude Toward Advertisement

\begin{tabular}{|c|c|c|c|c|c|}
\hline \multirow{2}{*}{ Variabel } & \multicolumn{2}{|c|}{$\begin{array}{c}\text { Unstandardzised } \\
\text { coefficients }\end{array}$} & $\begin{array}{c}\text { Standardized } \\
\text { coefficients }\end{array}$ & \multirow{2}{*}{ Signifikansi } \\
\cline { 2 - 4 } & $\mathrm{B}$ & Std. Error & Beta & & \\
\hline Constant & 8,829 & 1,380 & & & \\
\hline Kredibilitas SMI & 0,454 & 0,074 & 0,528 & 6,148 & 0,000 \\
\hline
\end{tabular}


We can see the significance value of the influence of SMI's credibility on attitude toward advertisement. From table 9., it can be concluded that significance value of the influence of SMI's credibility on attitude toward advertisement is 0,000 or below 0,05 . This implies that Gisella's credibility as SMI influence attitude toward advertisement of casual fashion. This result is in line with research conducted by Sallam (2011), Aziz et al. (2013), Rahmah (2015), Apejoye (2013), and Isosuo (2016) who found that SMI's credibility influence attitude toward advertisement.

Table 10. Influence of SMI's Credibility on Purchase Intention

\begin{tabular}{|c|c|c|c|c|c|}
\hline \multirow{2}{*}{ Variabel } & \multicolumn{2}{|c|}{$\begin{array}{c}\text { Unstandardzised } \\
\text { coefficients }\end{array}$} & $\begin{array}{c}\text { Standardized } \\
\text { coefficients }\end{array}$ & \multirow{2}{*}{ Signifikansi } & \\
\cline { 2 - 4 } & $\mathrm{B}$ & Std. Error & Beta & & \\
\hline Constant & 1,942 & 1,471 & & & \\
\hline Kredibilitas SMI & 0,540 & 0,079 & 0,570 & 6,864 & 0,000 \\
\hline
\end{tabular}

Table 10., showed that significance value of the influence of SMI's credibility on purchase intention value is 0,000 or below 0,05 . This implies that Gisella's credibility as SMI influence purchase intention of casual fashion. This result is in line with research conducted by Arsinta and Purnami (2015), Bhakar and Dubey (2015), Rachmatet al. (2016), Setyawati and Rosiana (2018) and Harly and Octavia (2014) who also found that SMI's credibility influence purchase intention.

Table 11. Influence of Customer Perceived Value on Purchase Intention

\begin{tabular}{|c|c|c|c|c|c|}
\hline \multirow{2}{*}{ Variabel } & \multicolumn{2}{|c|}{$\begin{array}{c}\text { Unstandardzised } \\
\text { coefficients }\end{array}$} & $\begin{array}{c}\text { Standardized } \\
\text { coefficients }\end{array}$ & \multirow{2}{*}{ S } & Signifikansi \\
\cline { 2 - 4 } & $\mathrm{B}$ & Std. Error & Beta & & \\
\hline Constant & 0,894 & 1,263 & & & \\
\hline $\begin{array}{c}\text { Customer } \\
\text { Perceived Value }\end{array}$ & 0,718 & 0,081 & 0,666 & 8,848 & 0,000 \\
\hline
\end{tabular}


As can be seen in table 11., the significance value of the influence of customer perceived value on purchase intention is 0,000 or below 0,05 . This implies that customer perceived value of casual fashion influence purchase intention. This result is in line with research conducted by Arsinta and Purnami (2015), Hansudoh (2012), Harly and Octavia (2014), Chi et al. (2011) who found that customer perceived value influence purchase intention.

Table 12. Influence of Attitude Toward Advertisement on Purchase Intention

\begin{tabular}{|c|c|c|c|c|c|}
\hline \multirow{2}{*}{ Variabel } & \multicolumn{2}{|c|}{$\begin{array}{c}\text { Unstandardzised } \\
\text { coefficients }\end{array}$} & $\begin{array}{c}\text { Standardized } \\
\text { coefficients }\end{array}$ & \multirow{2}{*}{ Signifikansi } & \\
\cline { 2 - 4 } & $\mathrm{B}$ & Std. Error & Beta & & \\
\hline Constant & 2,163 & 1,659 & & & \\
\hline $\begin{array}{c}\text { Attitude toward } \\
\text { advertisement }\end{array}$ & 0,567 & 0,095 & 0,515 & 5,941 & 0,000 \\
\hline
\end{tabular}

Table 8., showed that significance value of the influence of attitude toward advertisement on purchase intention is 0,000 or below 0,05 . This implies that consumer's attitude toward advertisement of casual fashion endorsed by Gisella as SMI influence purchase intention. This result is in line with research conducted by Rahmah (2015), Setyawati and Rosiana (2018), Rochmania and Prabowo (2016) and Sallam (2011) who found that attitude toward advertisement influence purchase intention.

\section{Path Analysis}

Path analysis and causal step method were chosen to examine the mediation effect of customer perceived value and attitude toward advertisement on the influence of SMI's credibility on purchase intention.

Table 11. Influence of SMI's Credibility, Customer Perceived Value, and Attitude Toward Advertisement on Purchase Intention

\begin{tabular}{|c|c|c|c|}
\hline \multirow{2}{*}{ Variabel } & \multicolumn{2}{|c|}{$\begin{array}{c}\text { Unstandardzised } \\
\text { coefficients }\end{array}$} & $\begin{array}{c}\text { Standardized } \\
\text { coefficients }\end{array}$ \\
\cline { 2 - 4 } & $\mathrm{B}$ & Std. Error & Beta \\
\hline Constant & $-3,001$ & 1,526 & \\
\hline
\end{tabular}




\begin{tabular}{|c|c|c|c|}
\hline Kredibilitas SMI & 0,247 & 0,083 & 0,260 \\
\hline Customer Perceived Value & 0,498 & 0,093 & 0,462 \\
\hline SikapKonsumenTerhadapIklan & 0,158 & 0,096 & 0,143 \\
\hline
\end{tabular}

(1) $\mathrm{CPV}=7,129+0,507 \mathrm{KS}+0,862$

(2) $\mathrm{SK}=8,829+0,528 \mathrm{KS}+0,849$

(3) $\mathrm{MB}=-3,001+0,462 \mathrm{CPV}+0,143 \mathrm{SK}+0,260 \mathrm{KS}+0,686$

Keterangan:

KS = Kredibilitas SMI

$\mathrm{CPV}=$ Customer Perceived Value

SK = SikapKonsumenTerhadapIklan

MB = MinatBeli

From table 11., it can be seen from standardized coefficients of the influence of SMI’s credibility on purchase intention is 0,260 . The mediation effect of customer perceived value on the influence of SMI's credibility on purchase intention is $0,507 \times 0,462=0,234$. The mediation effect of attitude toward advertisement on the influence of SMI's credibility on purchase intention is $0,528 \times 0,143=0,076$. From this calculation, it can be said that direct effect of SMI's credibility on purchase intention is bigger than indirect effects (through customer perceived value or attitude toward advertisement). This indicates that the mediation effect of both mediating variables are partial. It means that there are other mediating variables that can mediate SMI’s credibility on purchase intention.

This result is in line with research conducted by Arsinta and Purnami (2015), Bhakar and Dubey (2015), Hansudoh (2012), and Wiryantari and Sukawati (2016) who also found that customer perceived value partially mediates the influence of SMI's credibility on purchase intention. The higher the perceived value of product, than the higher the consumer will consider to buy that product (casual fashion). Higher perceived value can be achieved through the use of SMI to endorse casual fashion, such as what Gisella did. 
Also, this result is in line with research conducted by Setyawati and Rosiana (2018), Stephanie (2013), and Goldsmith (2000) who found that attitude toward advertisement mediates the influence of SMI's credibility on purchase intention. This is due to the ability of Gisella as SMI who is attractive. Thus when she did the advertisement on casual fashion she endorsed, the positive attitude toward advertisement was created, therefore contributed to purchase intention.

\section{CONCLUSION AND IMPLICATION}

From the result above, it can be concluded that Gisella's credibility significantly influence purchase intention of casual fashion on female student of Soegijapranata Catholic University Semarang. Customer perceived value and attitude toward advertisement act as partial mediating variables on the influence of Gisella's credibility on purchase intention of casual fashion on female student of Soegijapranata Catholic University Semarang.

From the result above, the implication to the producer of casual fashion need to consider how to improve customer perceived value on casual fashion, since it has the biggest effect on purchase intention. SMI as endorser can also be considered as the way to improve customer perceived value. The producer also needs to take on consideration about how credible SMI they choose, since SMI's credibility also poses a great effect on customer perceived value. Through the use of credible SMI, the information of casual fashion will be said well thus the value of casual fashion will be higher. Furthermore, the use of credible SMI, can lead to positive attitude toward advertisement. If the producer uses SMI who is attractive, trusted, popular, and suitable to promote casual fashion, then the consumer will deeply feel about the advertisement, making them want to buy that product SMI endorsed. 


\section{REFERENCES}

Ambarwati, M., Sunarti, Mawardi, M.K. (2015). Pengaruh Citra MerekTerhadapMinatBeli (SurveiPadaMahasiswaUniversitasBrawijaya yang Menggunakan Pasta Gigi Pepsodent). JurnalAdministrasiBisnis (JAB) Vol. 25(1): 1-7.

Apejoye, Adeyanju. (2013). Influence of Celebrity Endorsement of Advertisement on Student's Purchase Intention. Mass Communication and Journalism.

AsosiasiPenyelenggaraJasa Internet Indonesia. (2016). SaatnyaJadiPokokPerhatianPemerintahdanIndustri. Diaksesdari: https://apjii.or.id/downfile/file/BULETINAPJIIEDISI05November2016.pdf

Arsinta, G.A., Purnami N.M. (2015). PeranPersepsiNilaidalamMemediasiPengaruhKredibilitas Celebrity Endorser padaNiatbeliProdukKosmetik Maybelline di Kota Denpasar. JurnalManajemen, StrategiBisnisdanKewirausahaanVol. 9 (2): 123-134.

Ari, Ezgi. Nasir, Aslihan. (2014). A Critical Review of Online Consumers' Purchase Intentions. Advances in Business-Related Scientific Research Conference 2014 in Venice (ABSRC 2014 Venice).

Asgarpour, Rasoul., Sulaiman Z., Hamid, A.B.A. (2015). A Review on Customer Perceived Value and Its Main Components. Global Journal of Business and Social Science Review vol. 1(2): 632-640.

Aziz, S., Ghani U., Niazi A. (2013). Impact of Celebrity Credibility on Advertising Effectiveness. Pakistan Journal of Commerce and Social Sciencesvol. 7(1): 107-127.

Baron, R.M., Kenny, D.A. (1986). The Moderator-Mediator Variable Distinction in Social Psychological Research: Conceptual, Strategic, and Statistical Considerations. Journal of Personality and Social Psychologyvol. 51(5): 1173-1182.

Bhakar, S., Dubey, A. (2015). Analysis of The Factors Affecting Customer's Purchase Intention: Mediating Role of Customer Knowledge and Perceived Value. Advances in Social Sciences Research Journal Vol. 2(1): 87-101.

Chi, H., Yeh H.R., Tsai C. (2011). The Influences of Perceived Value on Consumer Purchase Intention: The Moderating Effect of Advertising Endorser. Conference Proceeding.

DailySocial. (2017). APJII: PenetrasiPengguna Internet Indonesia Capai 143 Juta Orang.

Ferdinand, A. (2006). Strategic Pathways Toward Sustainable Competitive Advantage. Unpublished DBA. Theses. Australia.

Freberg, K., Graham K., McGughey, K. \&Freberg, L.A. (2011). Who are the social media influencers? A study of public perceptions of personality. Public Relations Review vol. 37(1): 90-92.

Gashi, Linda. (2017). Social Media Influencers -Why We Cannot Ignore Them. Bachelor Thesis of Science in Business Administration: International Business and Marketing.

Ghozali, Imam. (2011). AplikasiAnalisis Multivariate dengan Program SPSS. Semarang: BadanPenerbitUniversitasDiponegoro.

Goldsmith, R.E., Lafferty B.A., Newell, S.J. (2000). The Impact of Corporate Credibility and Celebrity Credibility on Consumer Reaction to Advertisements and Brands. Journal of Advertising vol. 29 (3): 43-54. 
Gyda, D.S. (2017). The New Celebrities Working with Social Media Influencers. Thesis. Diaksesdari:

https://skemman.is/bitstream/1946/28392/1/gyda_the_new_celebrities_skemma.pdf

Hansudoh, S.A. (2012). Pengaruh Celebrity Endorsement terhadap Purchase Intention Melalui Perceived Value padaProduk Top Coffee di Surabaya. E-Journal UniverisitasKatolikWidya Mandala Surabaya vol. 1(5).

Harly, G. dan Octavia, D. (2014). Pengaruh Endorsement Fashion Blogger TerhadapMinatBeliMerekLokalpadaTahun 2013-2014 (StudiKasuspada Fashion Blogger Evita Nuh). JurnalManajemen Indonesia Vol. 14(2).

Indraswari, N.M., Pramudana, K.A.S. (2014). PengaruhKredibilitas Celebrity Endorser danKewajaranHargaTerhadapNiatbeliKonsumenWanitapada Online Shop ProdukPakaian. E-JurnalManajemenUniversitasUdayanaVol. 3(4): 938-955.

Instagram. (2018). About Us. Diaksesdari: https://instagram.com/about/us

Isosuo, Heli. (2016). Social Media Influencer Marketing. Bachelor Thesis. Diaksesdari: https://www.theseus.fi/bitstream/handle/10024/114035/thesisheliisosuo.pdf

Isparmo. (2016). Data StatistikPengguna Internet Indonesia Tahun 2016. Diaksesdari: http://isparmo.web.id/2016/11/21/data-statistik-pengguna-internet-indonesia-2016/

JarvisStore. (2016). 6 Produk yang Paling BanyakDijual Online. Diaksesdari: https://jarvisstore.com/artikel/6-produk-yang-paling-banyak-di-jual-online

LineToday. (2018). 7 Fashion Gisel yang SimpelTapiElegan, Cantik! Diaksesdarihttps://today.line.me/id/pc/article/7+Fashion+Gisel+yang+Simpel+Tapi+ Elegan+Cantik-2eVD8B

Maharani, Kumala., Totoatmojo. (2015). The Celebrity Endorser (Selebgram) Effect Toward Purchase Intention on Instagram Social Media. $3^{\text {rd }}$ AASIC: Sustainable Development of Asian Community.

McDougall, G.H. \& Levesque, T. (2000). Customer Satisfaction with Services: putting perceived value into the equation. Journal of Services Marketing vol. 14(5): 392-410.

Neves, A., Vieira, R., Mourao, F., \& Rocha, L. (2015). Quantifying Complementarity among Strategies for Influencers' Detection on Twitter1. Procedia Computer Science 51: 2435-2444.

Pakpahan, E.S.B. (2017). PengaruhKredibilitas Celebrity EndroserTerhadapKeputusanPembeliandanKepuasanKonsumen You-C 1000 Vitamin C di Kota Pekanbaru. JOMFekon vol. 4 no. 1: 721-734.

Rachmat, D.O.N., Ariyanti M., Zuliestiana D.A. (2016). Pengaruh Celebrity Endorser di Media Sosial Instagram dalamPromosiProduk Hijab terhadapMinatBeliKonsumen (StudiKasuspadaAkun Instagram @Zahratuljannahdan @joyagh).e-Proceeding of Management vol. 3(3): 2858-2865.

Rahmah, Nikmatur. (2015). IklanSensodynePadaSikapKonsumen PengaruhKredibilitas Endorser (TerhadapIklandanProduk) danMinatBeliKonsumen (StudipadaMahasiswaUniversitasBrawijaya Malang). JurnalEkonomiBisnisvol. 20(1): 1-53.

Rochmania, L., Prabowo, F. (2016). Pengaruh Celebrity Endorsement pada Instagram TerhadapMinatBeliProduk Mode Lokal (StudipadaSharenaGunawan). E-Proceeding of Management Vol. 3(2): 1103-1112.

Sallam, Methaq A.A. (2011). The Impact of Source Credibility on Saudi Consumer's Attitude toward Print Advertisement: The Moderating Role of Brand Familiarity. International 
Journal of Marketing Studies Vol. 3(4): 63-77. URL: http://dx.doi.org/10.5539/ijms.v3n4p63

Schiffman, Leon G., Kanuk, Leslie L. (2010). Consumer Behavior $10^{\text {th }}$ ed. Pearson Education.

Setyawat, Sri Murni; Rosiana, Monica. (2018). SikapKonsumenSebagaiMediasiPengaruhKredibilitasSelebritidan EWomTerhadapMinatBeli (StudiEmpirispadaKonsumen Bandung Makuta). Seminar Nasional dan Call for Paper Sustainable Competitive Advantage (SCA).

Shafiq, R., Raza, I. Zia-ur-Rehman, M. (2011). Analysis of the Factors Affecting Customer's Purchase Intention: The Mediating Role of Perceived Value. African Journal of Business Management Vol. 5(26): 10577-10585.

Shimp, T.A. (2003). PeriklananPromosi. Jakarta: Erlangga.

Smartinsights. (2016). Global Social Media Research Summary 2016. Diaksesdari: https://c.ymcdn.com/sites/www.productstewardship.us/resource/resmgr/Phone_Books /Global_Social_Media_Statisti.pdf

Sociabuzz. (2018). KupasTuntasTrenPemasaran "Endorse". The State of Influencer Marketing 2018 in Indonesia. Diaksesdari: https://www.labana.id/wpcontent/uploads/2018/03/The-State-of-Influencer-Marketing-2018-in-IndonesiaKupas-Tuntas-Tren-Pemasaran-Endorse.pdf

Stafford, M.R. Spears, N.E. Hsu, C. (2003). Celebrity Images in Magazine Advertisements: an Application of The Visual Rhetoric Model. Journal of Current Issue and Research in Advertising Vol. 25(2): 13-20.

Stephanie, Elysia. (2013). PengaruhKredibilitas Endorser TerhadapMinatBeliMelaluiSikapAtasIklanpadaProdukShampoL'oreal di Surabaya. JurnalIImiahMahasiswaUniversitas Surabaya Vol. 2 (2): 1-21.

Stephanie, E., Rumambi L.J., Kunto, Y.S. (2013). AnalisaPengaruh Rio DewantodanDonitaSebagai Celebrity Endorser TerhadapMinatBeliProdukAze Anarchy denganDaya Tarik IklandanEfekIklansebagaiVariabel Intervening. JurnalManajemenPemasaran Vol. 1 (2): 1-9.

Sudha, M. \& Sheena K. (2017). Impact of Influencers in Consumer Decision Process: the Fashion Industry. SCMS Journal of Indian Management: 14-30.

Szmigin, L. \&Piacentini, M. (2015). Consumer Behaviour (Vol. 1). Oxford: Oxford University Press.
Wangsa,
G.C.U.,
Ardani
I.G.

(2015).

PeranSikapPadaIklandalamMemediasiPengaruhKredibilitasAlGhazaliSebagai

Endorser TerhadapNiatBeli (StudiPadaProduk Men's Biore Cool Oil Clear di Kota Denpasar). E-jurnalmanajemen Vol. 4(3): 538-557. 